\title{
天然能量开发阶段的缝洞型油藏井间连通性分析
}

\author{
苏泽中 ${ }^{1}$ ，林加恩 ${ }^{1,2}$ ，柏明星 ${ }^{1}$ ，吴德胜 ${ }^{3}$ ，刘 亮 $^{4}$ ，朱建红 ${ }^{1}$
}

1) 西安石油大学石油工程学院, 陕西西安 710065；2) 西安石油大学西部低渗-特低渗油藏开发与

治理教育部工程研究中心，陕西西安 $710065 ； 3$ ）中国石油新疆油田分公司工程技术研究院，新疆克拉玛依 834000 ;

4) 中国石油天然气管道工程有限公司，河北廊坊 065000

摘 要: 研究碳酸盐岩缝洞型油藏天然能量开发阶段的采油井间连通关系对于后续实施注水开发、确 定注采对应关系和转注油井以及进行注水井位部署都具有重要意义. 由于静态法在矿场井间连通性分析中 存在不确定性，所以通常采用动态连通性分析井间连通情况. 本研究从生产动态数据入手，优选油压、流 压、产能和动液面 4 类动态数据，首先基于动态时间规整算法，设计井间连通识别方法进行采油井间连通 分析; 其次以采油井间的动态曲线相似度为基础，采用 “五标度改进层次分析-摘权法” 计算各动态曲线相 似度的指标权重; 最后建立天然能量开发阶段㖓洞型油藏的采油井间连通程度评价模型. 利用该模型对某 缝洞油藏进行实例分析, 其评价结果与已知的静态连通分析结果及生产动态特征一致性高, 验证了评价模 型的准确性. 该模型可以为天然能量开发阶段的缝洞型油藏采油井连通性分析提供参考.

关键词: 油田开发; 缝洞型油藏; 天然能量开发; 采油井连通程度; 生产动态数据; 动态时间规整算 法; 五标度改进层次分析一摘权法; 权重分析

中图分类号：TE344 文献标志码：A doi: 10.3724/SP. J. 1249.2020.06645

\section{Inter-well connectivity analysis in carbonate fracture-vuggy reservoir in natural energy development stage}

\section{SU Zezhong' ${ }^{1}$, LIN Jiaen ${ }^{1,2}$, BAI Mingxing' ${ }^{1}$ WU Desheng ${ }^{3}$, LIU Liang ${ }^{4}$, and ZHU Jianhong ${ }^{1}$}

1) College of Petroleum Engineering, Xi'an Shiyou University, Xi'an 710065, Shaanxi Province, P. R. China

2) MOE Engineering Research Center of Development \& Management of Western Low \& Ultra-Low Permeability Oilfield, Xi'an Shiyou University, Xi'an 710065, Shaanxi Province, P. R. China

3 ) Engineering Technology Research Institute, Petro China Xinjiang Oilfield Company, Karamay 834000, Xinjiang, P. R. China 4) China Petroleum Pipeline Engineering Co. Ltd. (Engineering), Langfang 065000, Hebei Province, P. R. China

\footnotetext{
Abstract: It is of great importance to study the inter-well connectivity in the carbonate fracture-vuggy reservoirs during the stage of natural energy development, for the subsequent implementation water-flooding development, injection wells deployment, corresponding injection-production wells relationship characterization, and the choice of

Received: 2020-07-11; Accepted: 2020-08-31

Foundation: Key R \& D Program of Shaanxi Province (2019ZDLGY11-04)

Corresponding author: Professor LIN Jiaen. E-mail: jn5000@126.com

Citation: SU Zezhong, LIN Jiaen, BAI Mingxing, et al. Inter-well connectivity analysis in carbonate fracture-vuggy reservoir in natural energy development stage $[\mathrm{J}]$. Journal of Shenzhen University Science and Engineering, 2020, 37(6) : 645-652. (in Chinese)
} 
production wells transfer injection wells. In view of the uncertainty of static geological analysis, the dynamic analysis method is usually more preferred to study the inter-well connectivity. In this work, four types of dynamic data are selected for analysis, including tubing pressure, formation flowing pressure, oil capacity and working fluid level. An inter-well connectivity recognition method is then designed in use of dynamic time warping (DTW) algorithm to analyze production wells connectivity. The five-scale IAHP and entropy method is also used to calculate the index weight based on the dynamic curve similarities of production wells. Followed by, production well connectivity evaluation model is established for carbonate fracture-vuggy reservoir in the natural energy development stage. A fracture-vuggy reservoir example is analyzed, and the result shows an excellent matching with the existing results of static geological connectivity and production dynamic characteristics, which verifies the accuracy of the evaluation model. This model provides valuable insight on the production wells connectivity in carbonate fracture-vuggy reservoirs.

Key words : oil field development; fracture-vuggy reservoir; natural energy development; production wells connectivity; production dynamic data; dynamic time warping algorithm; five-scale improved AHP and entropy weight method; weight analysis

缝洞型碳酸盐岩油藏是指在碳酸盐岩圈闭中形 成的原油聚集，其储渗空间为裂缝和溶洞，基质岩 石不具备渗透能力 ${ }^{[1-2]}$. 塔河油田位于中国塔里木 盆地阿克库勒凸起北部, 是典型的缝洞型碳酸盐岩 油藏. 多期 “构造-岩溶-成藏” 作用形成的复杂缝 洞系统导致塔河油田具有极强的非均质性和复杂的 油水关系，对油藏开发效果产生了极大影响 ${ }^{[3]}$. 塔 河油田以 “缝洞单元” 作为基础开发单元，因为采 用 “点状注水 + 不规则井网” 的注水开发方式，所 以需要对天然能量开发阶段的采油井进行进间连通 性分析，为后续注水开发提供技术支持.

对于缝洞型油藏井间连通性的研究进展，可以 根据缝洞单元的开发阶段进行分类叙述. 对于注水 开发阶段的缝洞单元, 主要研究注采井之间的连通 关系, 研究方法包括静态法和动态法. 静态法采用 地震属性反演储层地质特征进行连通性分析, 其本 质反映的是地层骨架的连通情况，在实际应用中静 态分析具有误差和不确定性，因此矿场更多采用动 态生产数据进行连通性分析. 动态法主要包括测试 分析 ${ }^{[4-6]}$ 、生产动态分析 ${ }^{[7-11]}$ 和数学模型反演 ${ }^{[12-17]}$ 等, 测试和生产动态分析属于定性分析, 数学模型 反演则属于定量表征. 对于处在天然能量开发阶段 的缝洞单元, 主要研究采油井之间的连通关系, 矿 场通过静态地质分析、干扰测试和井间生产动态等 手段进行定性分析，而采油井之间的连通性定量评 价研究较少.

针对缝洞型油藏天然能量开发阶段采油井间连 通性定量评价问题, 采用油藏生产动态数据, 建立 了基于多指标权重分析法的采油井间连通性定量评
价模型, 以期为天然能量开发阶段采油井间连通性 定量评价提供可借鉴的思路和方法.

\section{1 采油井连通分析及定量计算}

\section{1 评价指标优选}

缝洞单元是被不渗透基岩包围, 内部由裂缝、 溶蚀空洞和溶洞等交错组合形成的流动单元 ${ }^{[2]}$, 具 有独立的压力和水动力系统. 油藏开发过程中压 力、生产数据和动液面分别反映缝洞单元内部压力 系统、水动力系统和能量系统的变化. 选择 $\mathrm{A}$ 单元 的 15 口采油井自喷期内前 3 个月的生产数据点 （流压、油压、套压、产液量、产油量和动液面） 进行线性相关性分析，得到油压和套压的线性相关 方程为 $y_{1}=3.011 x_{1}-50.769, R^{2}=0.963$; 产液量 和产油量的线性相关方程为 $y_{2}=0.964 x_{2}+1.767$, $R^{2}=0.997$ (含水率即油井采出液中产出水的质量 分数, 低于 $2 \%), y_{3}=0.735 x_{3}+3.351, R^{2}=$ 0.791 (含水率高于 $2 \%$ ). 结果表明, 油压和套压、 产液量和产油量的相关性高. 因为油压能够反映油 井能量的变化, 而套压只能反映环空压力的变化, 所以选择流压、油压、产油量和动液面进行采油井 间连通定量评价. 结合矿场实际将产油量数据修正 为产能数据, 即

$$
q_{0}=Q_{0} / t
$$

其中, $q_{0}$ 为油井产油能力 (单位: $\mathrm{t} / \mathrm{d}$ ); $Q_{0}$ 为油井 阶段实际产油量 (单位: $\mathrm{t}$ ) ; $t$ 为对应的实际生产天 数 (单位: $d$ ). 


\section{2 连通判定及动态曲线相似度计算}

处于同一缝洞单元中且相互连通的采油井, 在 同时间段的生产过程中会表现出相似的动态变化特 征，这正是进行采油井组连通判定的依据，如图 1 所示. 通过计算两口采油井动态曲线（流压、油 压、产油量和动液面）的相似程度，即可定量判断 两口采油井的连通程度.

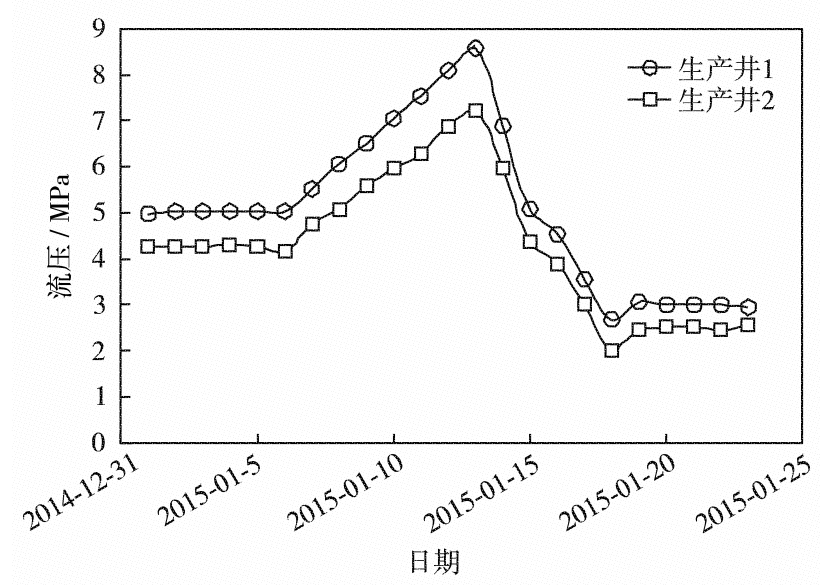

图 1 采油井间连通性定性分析原理

Fig. 1 Qualitative analysis of production wells connectivity

利用动态时间规整 ( dynamic time warping, DTW）算法可以计算曲线的相似程度，该算法采用 动态规划计算时间序列间的最优映射来表示序列间 的相似程度 ${ }^{[18]}$. 将采油井的动态曲线看作是具有 一定长度的时间序列，通过计算两个时间序列间的 DTW 值，可以定量表征时间序列间的相似程度. DTW 值越小，两个时间序列间的相似程度越高， 表明两口采油井的连通性越好; 反之 DTW 值越大， 连通性越差.

具体计算过程如下：已知两个长度为 $m$ 和 $n$ 的 时间序列 $A(t)=\left\{A_{1}, A_{2}, \cdots, A_{m}\right\}$ 和 $B(t)=\left\{B_{1}\right.$, $\left.B_{2}, \cdots, B_{n}\right\}$, 按照时间位置将其排序构建距离矩阵 $C_{m \times n}$, 元素 $c_{i j}=\sqrt{\left(A_{i}, B_{j}\right)^{2}}$, 为 2 个时间点间的距 离. 在 $C_{m \times n}$ 中, 把一组相邻的矩阵元素集合成为弯 曲路径, 记为 $W=\left\{w_{1}, w_{2}, \cdots, w_{k}\right\}, W$ 的第 $k$ 个元 素 $w_{k}=\left(c_{i j}\right)_{k}$, 路径需要满足: (1) $\max \{m, n\}<K \leqslant$ $m+n-1$; (2) $w_{1}=c_{11}, w_{k}=c_{m n}$; (3) $w_{k}=c_{i j}, w_{k-1}=$ $c_{i^{\prime} j^{\prime}}$, 其中, $i-i^{\prime} \geqslant 0,0 \leqslant j-j^{\prime} \leqslant 1$, 则

$$
D[A(t), B(t)]=\min \frac{1}{K} \sum_{i=1}^{K} W_{i}
$$

DTW 算法采用动态规划思想寻找具有最小弯 曲代价的最佳路径, 即

$$
\begin{aligned}
& D(1,1)=c_{11} \\
& D\left(A(t)_{i}, B(t)_{j}\right)=c_{11}+ \\
& \min \left\{\begin{array}{l}
D\left(A(t)_{i-1}, B(t)_{j-1}\right), \\
D\left(A(t)_{i}, B(t)_{j-1}\right), \\
D\left(A(t)_{i-1}, B(t)_{j}\right)
\end{array}\right\}=c_{11}+ \\
& \min \left(c_{i-1, j-1}, c_{i, j-1}, c_{i-1, j}\right)
\end{aligned}
$$

其中, $i=2,3, \cdots, m ; j=2,3, \cdots, n$. 通过距离矩 阵 $C_{m \times n}$ 中弯曲路径的最小累加值 $D(m, n)$ 计算 DTW 值.

\section{3 阈值确定及井间连通识别}

\subsection{1 阈值确定}

缝洞单元内部的采油井并非全部连通, 因此, 计算出两条动态曲线的相似度后, 需要确定阈值判 断两口井是否连通. 以油压为例说明阈值确定 方法:

1) 计算 $D_{y}$ (两口采油井油压曲线的 DTW 值) 的极差 $d_{y}$,

$$
d_{y}=D_{y \max }-D_{y \min }
$$

其中, $D_{\text {max }}$ 为缝洞单元中油压曲线 DTW 值的最大 值; $D_{y \min }$ 为缝洞单元中油压曲线 DTW 值的最小值.

2) 采用 Sturgers 经验公式计算组距,

$$
f=1+3.322 \lg N
$$

其中, $N$ 为缝洞单元内所有油压曲线 DTW 值数量.

3 ) 选择 $D_{y}$ 数据量分布最多的两个区间, 计算 $D_{y}$ 的平均值 $\bar{D}_{y}$ 确定为阈值. 若两口采油井 $D_{y}$ 值小 于 $\bar{D}_{y}$, 则两井连通; 否则, 不连通.

\subsection{2 井间连通识别}

结合实际的矿场生产情况，充分考虑关停井、 生产时间不对应等问题，设计了采油井间连通识别 方法, 实现计算机的自动识别, 工作流程如图 2 所 示, 并以油压为例进行说明.

1) 选择缝洞单元, 提取采油井油压数据.

2) 确定各采油井自喷期, 自喷期确定规则如 下：投产开始后，油嘴大于 0 且没有洜挂深度，自 喷期时间小于含水时间，若油井未见水则当前时间 为自喷期. 目的是消除机抽和注水对数据的影响, 确保驱动能量来自油藏本身.

3 ）计算各井自喷期平均油压，平均油压大于 $1.5 \mathrm{MPa}$ 时, 进人步骤 4); 否则, 剔除该井, 退回 步骤 1). 目的是识别待分析采油井, 并且消除平 均流压的异常值对平均流压倍数的影响. 
4）选择相同的自喷期时间段，计算两口采油 井的平均流压倍数，平均流压倍数小于 2 倍时，进 人步骤 5 ). 目的是消除两口井因为油压变化范围 较大对曲线相似度计算的影响, 确保相似度计算和 井间连通判断，在合理误差范围内.

5 ）计算动态曲线的相似度.

6）进行阈值计算，确定两口采油井的连通情 况. 当 $D_{y} \leqslant \bar{D}_{y}$ 时, 表明两口采油井连通; 否则, 不连通.

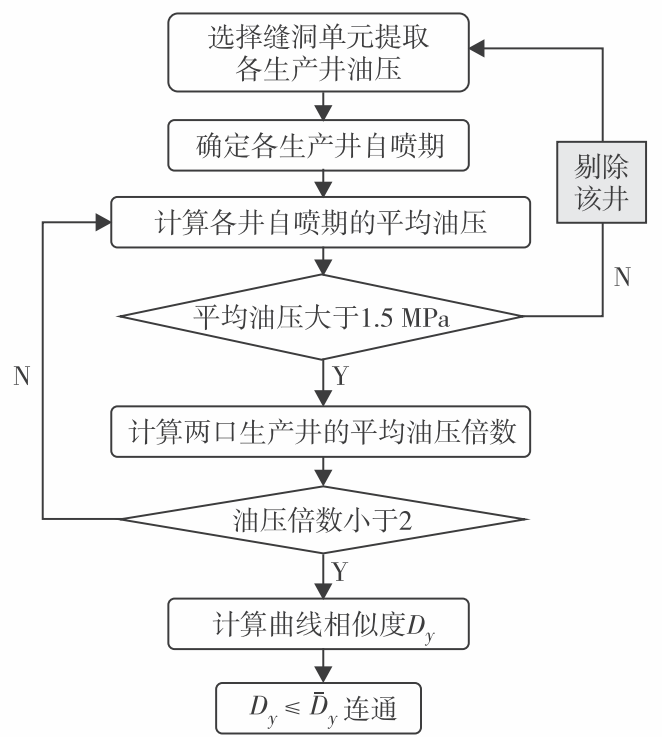

图 2 井间连通识别流程

Fig. 2 The workflow of inter-well connectivity identification

流压进行井间连通识别时，采用流压截距倍数 识别待分析井组, 截距倍数为 2 ; 产能进行连通识 别时, 采用平均产能倍数识别待分析井组, 平均产 能倍数为 2 ; 动液面进行连通识别时，采用自喷期 油压差识别待分析井组, 自喷期油压差为 $5 \mathrm{MPa}$.

\section{4 井间连通程度定量表征}

如果直接采用单一指标进行井间连通程度定量 分析，会因为评价的局限性导致井间连通情况不能 被准确描述. 例如, $A 、 B$ 井间通过动液面可以进行 井间连通情况定量表征，但是 $C 、 D$ 井未测得动液 面，则不能通过动液面进行井间连通定量表征. 实 际上, 采用其他动态数据也可以定量表征 $C 、 D$ 井 之间的连通程度. 因此本研究从多重指标评价思路 出发, 建立采油井间连通程度定量计算模型.

\subsection{1 多指标权重计算}

基于改进的五标度层次分析法 ${ }^{[19]}$ 计算指标的 权重，并采用熵权法 ${ }^{[20]}$ 对评价指标进行修正.
五标度层次分析法步骤: (1) 构建层次结构模 型, 目的层为井间连通系数 $I_{\mathrm{C}}$, 方案层包括油压曲 线相似度 $D_{\mathrm{y}}$ 、流压曲线相似度 $D_{1}$ 、产能曲线相似度 $D_{\mathrm{c}}$ 和动液面曲线相似度 $D_{\mathrm{d}}$, 如图 3 所示; (2) 根据 层次结构模型中方案层的评价指标, 确定比较矩阵 $X_{4 \times 4}$, 比较矩阵赋值原则如表 1 所示, 并计算重要 性排序指数 $R$; (3) 根据式 (7) 和 (8) 对比较矩阵进行 变换, 得到判断矩阵 $U_{4 \times 4}$; (4) 根据式 (9) 对判断矩 阵 $\boldsymbol{U}_{4 \times 4}$ 进行变换，确定反对称矩阵 $\boldsymbol{H}_{4 \times 4}$; (5) 根据式 (10) 对反对称矩阵 $\boldsymbol{H}_{4 \times 4}$ 进行变换, 确定最优传递 矩阵 $\boldsymbol{G}_{4 \times 4}^{*}$; (6) 采用方根法求解 $\boldsymbol{G}_{4 \times 4}^{*}$ 的特征向量, 求 解指标初始权重 $W_{c}$.

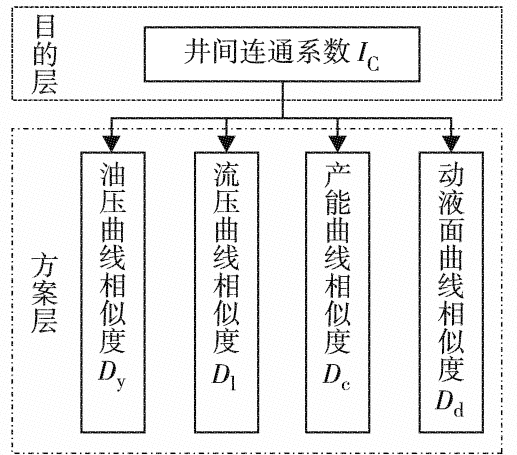

图 3 层次结构模型示意图

Fig. 3 Schematic diagram of hierarchical structure model

表 1 五标度层次分析法的赋值原则

Table 1 Assignment principles of five-scale AHP

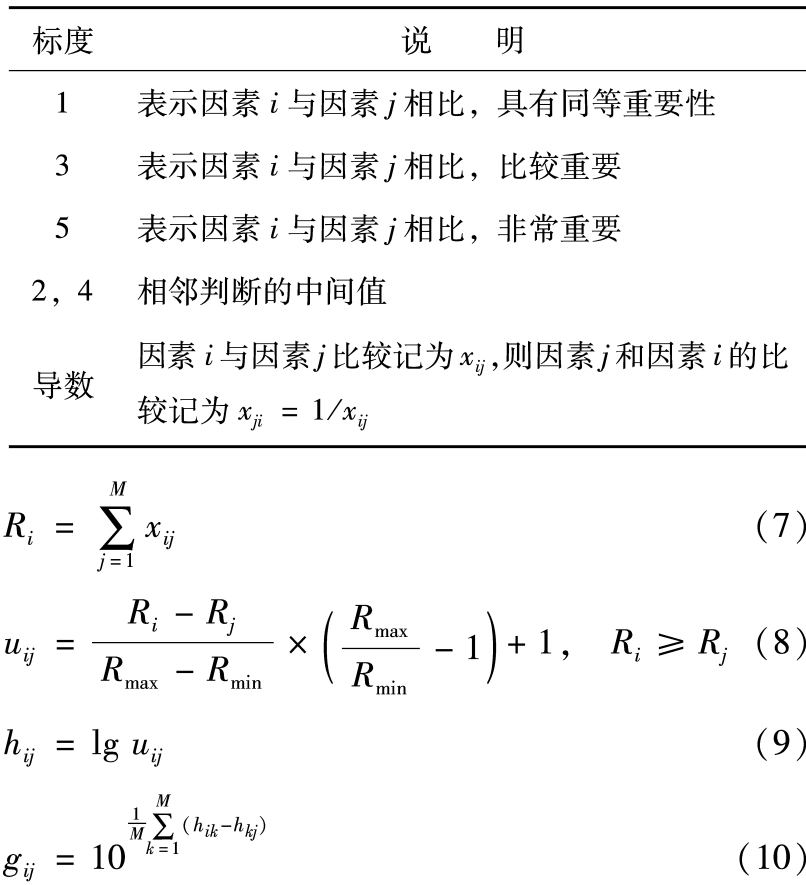


其中, 本研究 $M=4 ; i, j, k=1,2,3,4 ; x_{i j}$ 为比较 矩阵 $X_{4 \times 4}$ 的元素; $R_{i}$ 为 $X_{4 \times 4}$ 的行重要性排序指数; $R_{j}$ 为比较矩阵 $X_{4 \times 4}$ 的列重要性排序指数; $R_{\text {min }}$ 和 $R_{\text {max }}$ 分别为最大、最小重要性排序指数; $u_{i j}$ 为判断 矩阵 $\boldsymbol{U}_{4 \times 4}$ 的元素; $h_{i j}, h_{i k}$ 和 $h_{k j}$ 为反对称矩阵 $\boldsymbol{H}_{4 \times 4}$ 的 元素. $g_{i j}$ 为为最优传递矩阵 $\boldsymbol{G}_{4 \times 4}^{*}$ 的元素; 需要指 出, 当 $R_{i} \leqslant R_{j}$ 时, $u_{i j}$ 取式 (8) 计算值的倒数.

采用熵权法对五标度层次分析法得到的权重 $W_{\mathrm{c}}$ 进行修正. 根据判断矩阵 $\boldsymbol{U}_{4 \times 4}$ 计算各指标的熵 值 $T_{i}$,

$$
T_{i}=-(\ln M)^{-1} \sum_{j=1}^{M} f_{i j} \ln f_{i j}, \quad i=1,2, \cdots, M
$$

其中, $f_{i j}=R_{i j} / \sum_{j=1}^{M} R_{i j}$, 当 $f_{i j}=0$ 时, $f_{i j} \ln f_{i j}=0$.

采用熵权法计算的初始权重 $W_{\mathrm{si}}$ 对五标度层次 分析法计算的初始权重 $W_{c i}$ 进行修正, 得到最终权 重 $W$ 为

$$
W=\frac{W_{\mathrm{c} i} W_{\mathrm{s} i}}{\sum_{i=1}^{M} W_{\mathrm{c} i} W_{\mathrm{s} i}}, \quad i=1,2, \cdots, M
$$

\subsection{2 井间连通程度计算模型}

基于已确定的各指标权重，得到采油井间连通 系数 $I_{\mathrm{C}}$ 的计算公式为

$$
I_{\mathrm{C}}^{J}=\sum_{q=1}^{4}\left[2-\frac{D_{q}^{J}}{\max \left(D_{q}\right)-\min \left(D_{q}\right)}\right] \times \lambda_{q}
$$

其中, $q$ 为动态曲线相似度的种类, $q=1 \sim 4$, 分别 代表油压、流压、产能和动液面曲线相似度; $J$ 为 缝洞单元内部某类动态曲线相似度的数量, $D_{q}^{J}$ 为缝 洞单元内某两口采油井 (数量 $J$ ) 的 $q$ 类动态曲线相 似度; $\max \left(D_{q}\right)$ 为缝洞单元内某类动态曲线相似度 最大值; $\min \left(D_{q}\right)$ 为缝洞单元内某类动态曲线相似 度最小值; $\lambda_{q}$ 为 $q$ 类动态曲线相似度指标的权重.

\section{2 实例分析}

$A$ 缝洞单元位于塔河油田某区, 单元面积超过 $16 \mathrm{~km}^{2}$, 地质储量大于 $1500 \times 10^{4} \mathrm{t}$, 单元内采油井 15 口. 自 2008 年 9 月开始进行全面开发, 历经上 产阶段和递减阶段，截止至 2017 年底全面进人稳 产阶段, 综合含水率 $7.5 \%$, 采出程度为 $7.3 \%$ 。 单元处于天然能量开采阶段, 为了对后续注水开 发、注水井位部署和选择转注油井提供技术支持,
需要进行单元内部采油井间动态连通性分析.

首先，采用设计的井间连通识别方法对 $\mathrm{A}$ 单元 内所有采油井进行单指标连通识别. 油压、流压、 产能和动液面曲线的相似度阈值分别为: $\bar{D}_{\mathrm{y}}=$ $1.000, \bar{D}_{1}=2.330, \bar{D}_{\mathrm{c}}=9.770, \bar{D}_{\mathrm{d}}=131.600$, 得 到的 $\mathrm{A}$ 单元内部采油井间连通情况, 如表 2 .

\begin{tabular}{|c|c|c|}
\hline 连通分类 & 连通井组 & 曲线相似度 \\
\hline \multirow{3}{*}{ 动液面 } & A12-2 和 A258X & 48.870 \\
\hline & $\mathrm{A} 258 \mathrm{X}$ 和 $\mathrm{A} 209 \mathrm{CH}$ & 102.970 \\
\hline & $\mathrm{A} 253 \mathrm{X}$ 和 $\mathrm{A} 209 \mathrm{CH}$ & 114.490 \\
\hline 流 压 & A227X 和 A246X & 0.140 \\
\hline \multirow{5}{*}{ 油 压 } & A12-2 和 A12-6 & 0.470 \\
\hline & A12-2 和 A209CH & 0.190 \\
\hline & A12-2 和 A258X & 0.220 \\
\hline & A12-6 和 A209CH & 0.780 \\
\hline & A227X 和 A246X & 0.050 \\
\hline \multirow{6}{*}{ 产 能 } & A12-2 和 A12-6 & 2.300 \\
\hline & A12-2 和 A209CH & 6.350 \\
\hline & A12-6 和 A209CH & 4.280 \\
\hline & $\mathrm{A} 253 \mathrm{X}$ 和 $\mathrm{A} 209 \mathrm{CH}$ & 6.090 \\
\hline & $\mathrm{A} 253 \mathrm{X}$ 和 $\mathrm{A} 258 \mathrm{X}$ & 9.840 \\
\hline & A227X 和 A246X & 5.980 \\
\hline
\end{tabular}

表 $2 \mathrm{~A}$ 单元采油井间连通情况

Table 2 Production wells connectivity in unit A

其次，采用 “五标度层次分析-熵权法” 计算 评价指标的权重. 根据表 1 确定的评价指标比较矩 阵 $X_{4 \times 4}$, 计算重要性排序指数 $R$, 其中, $R_{\text {max }}=$ $10.000, R_{\min }=2.830$, 如表 3 ; 根据式 (8) 确定评价 指标的判断矩阵 $U_{4 \times 4}$, 如表 4 ; 根据式 (9) 确定评价 指标的反对称矩阵 $\boldsymbol{H}_{4 \times 4}$ 如表 5 ; 并确定评价指标的 最优传递矩阵 $G_{4 \times 4}^{*}$ 如表 6 ; 利用方根法求解矩阵 $G_{4 \times 4}^{*}$ 的特征向量为 $\xi=(1.210,0.565,2.428$, $0.602)$, 计算得到评价指标的初始权重为 $W_{\mathrm{c}}=(0.250,0.120,0.500,0.130)$. 采用熵权法对 $W_{\mathrm{c}}$ 进行修正, 将判断矩阵 $U_{4 \times 4}$ 采用极值法进行标 准化处理后采用式 (11) 计算得到熵权法初始权重为 $W_{\mathrm{s}}=(0.260,0.220,0.300,0.220)$, 最后根据式 (12) 计算得到修正后的指标权重为 $W=(0.240$, $0.100,0.550,0.110$ ). 
表 3 评价指标的比较矩阵

Table 3 Comparison matrix of evaluation index

\begin{tabular}{cccccc}
\hline & $D_{\mathrm{y}}$ & $D_{\mathrm{l}}$ & $D_{\mathrm{c}}$ & $D_{\mathrm{d}}$ & $R$ \\
\hline$D_{\mathrm{y}}$ & 1.000 & 2.000 & 0.500 & 2.000 & 5.500 \\
$D_{1}$ & 0.500 & 1.000 & 0.333 & 1.000 & 2.830 \\
$D_{\mathrm{c}}$ & 2.000 & 3.000 & 1.000 & 4.000 & 10.000 \\
$D_{\mathrm{d}}$ & 0.500 & 1.000 & 0.250 & 1.000 & 2.750 \\
\hline
\end{tabular}

表 4 评价指标的判断矩阵

Table 4 Judgment matrix of evaluation index

\begin{tabular}{ccccc}
\hline & $D_{\text {y }}$ & $D_{1}$ & $D_{\text {c }}$ & $D_{\text {d }}$ \\
\hline$D_{\text {y }}$ & 1.000 & 1.972 & 0.379 & 2.013 \\
$D_{1}$ & 0.507 & 1.000 & 0.277 & 1.033 \\
$D_{\mathrm{c}}$ & 2.639 & 3.611 & 1.000 & 3.640 \\
$D_{\mathrm{d}}$ & 0.497 & 0.968 & 0.275 & 1.000 \\
\hline
\end{tabular}

表 5 评价指标的反对称矩阵

Table 5 Anti-symmetric of evaluation index

\begin{tabular}{ccccc}
\hline & $D_{\text {y }}$ & $D_{1}$ & $D_{\text {c }}$ & $D_{\text {d }}$ \\
\hline$D_{\text {y }}$ & 0.000 & 0.295 & -0.421 & 0.304 \\
$D_{1}$ & -0.295 & 0.000 & -0.558 & 0.014 \\
$D_{\text {c }}$ & 0.421 & 0.558 & 0.000 & 0.561 \\
$D_{\text {d }}$ & -0.304 & -0.014 & -0.561 & 0.000 \\
\hline
\end{tabular}

表 6 判断矩阵的最优传递矩阵

Table 6 Optimal transfer matrix of judgment matrix

\begin{tabular}{ccccc}
\hline & $D_{\text {y }}$ & $D_{1}$ & $D_{\text {c }}$ & $D_{\text {d }}$ \\
\hline$D_{\text {y }}$ & 1.000 & 2.551 & 0.457 & 1.838 \\
$D_{\mathrm{l}}$ & 0.392 & 1.000 & 0.254 & 1.023 \\
$D_{\text {c }}$ & 2.190 & 3.933 & 1.000 & 4.026 \\
$D_{\mathrm{d}}$ & 0.544 & 0.977 & 0.248 & 1.000 \\
\hline
\end{tabular}

最后，采用式 (13) 计算 $\mathrm{A}$ 单元内部采油井间 连通系数 $I_{\mathrm{C}}$, 并绘制井间连通示意图，如表 7 和图 4. 计算得到 $\mathrm{T}$ 单元内部 $\mathrm{A} 246 \mathrm{X}-\mathrm{A} 227 \mathrm{X}$ 的井间连 通系数最大为 1.512 , 地震属性反演显示 $\mathrm{A} 246 \mathrm{X}$ 井 和 $\mathrm{A} 227 \mathrm{X}$ 井位于单元内部断裂带的溶蚀段内, 表 明该两口井在静态地质分析中是连通的，如图 5; 此外对两口井生产曲线分析, 发现 A246X 井和 $\mathrm{A} 227 \mathrm{X}$ 井的流压和油压曲线的动态变化具有高度一 致性, 产能曲线在某些时间段内也表现出相同的变 化趋势，表明 A246X 和 A227X 井的连通情况是最 好的, 同时也对式 (13) 的准确性进行了验证, 如图 6 至图 8. 对于后续的注水开发而言，建议可以对 $\mathrm{A} 258 \mathrm{X}$ 井进行转注或者在图 4 中三角形区域内钻注 水井进行注水开发达到注水增油效果.
表 $7 \mathrm{~A}$ 单元采油井间连通井组及连通系数

Table 7 Production wells connectivity coefficient of A unit

\begin{tabular}{cc}
\hline 连通井组 & $I_{\mathrm{C}}$ \\
\hline $\mathrm{A} 12-2$ 和 $\mathrm{A} 12-6$ & 1.442 \\
$\mathrm{~A} 12-2$ 和 $\mathrm{A} 209 \mathrm{CH}$ & 1.239 \\
$\mathrm{~A} 12-2$ 和 $\mathrm{A} 258 \mathrm{X}$ & 0.643 \\
$\mathrm{~A} 12-6$ 和 $\mathrm{A} 209 \mathrm{CH}$ & 1.196 \\
$\mathrm{~A} 227 \mathrm{X}$ 和 $\mathrm{A} 246 \mathrm{X}$ & 1.512 \\
$\mathrm{~A} 253 \mathrm{X}$ 和 $\mathrm{A} 209 \mathrm{CH}$ & 0.952 \\
$\mathrm{~A} 253 \mathrm{X}$ 和 $\mathrm{A} 258 \mathrm{X}$ & 0.550 \\
$\mathrm{~A} 258 \mathrm{X}$ 和 $\mathrm{A} 209 \mathrm{CH}$ & 0.109 \\
\hline
\end{tabular}

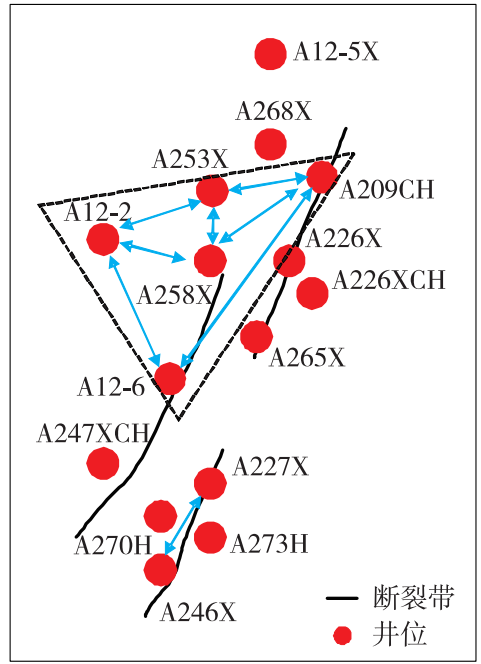

图 4 A 单元采油井间连通示意图

Fig. 4 (Color online) Production wells connectivity diagram of unit $A$

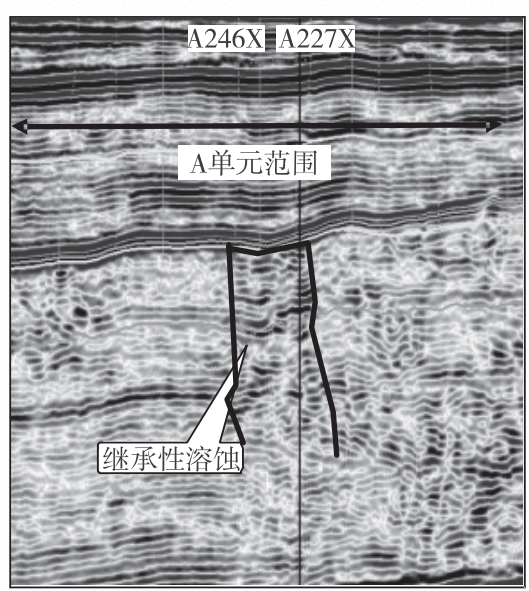

图 $5 \mathrm{A246X}$ 和 A227X 井地震属性反演图

(据中石化西北油田)

Fig. 5 Inversion map of seismic attributes of A246X and A227

(According to the Northwest Oilfield, Sinopec) 


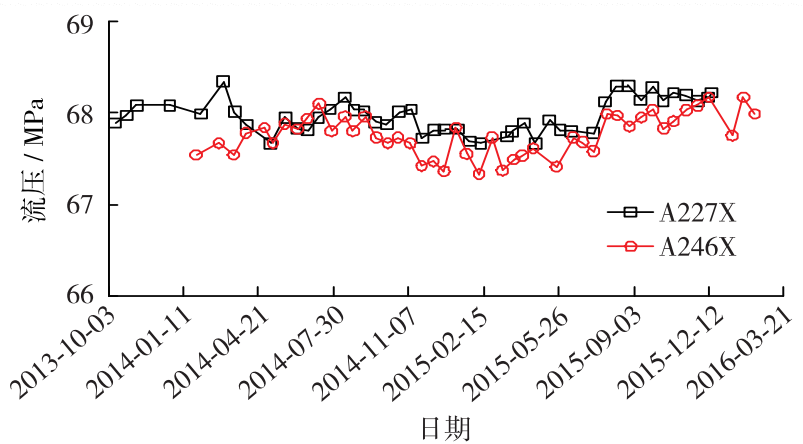

图 $6 \mathrm{A227X}$ 和 $\mathrm{A246X}$ 井流压动态曲线

Fig. 6 (Color online) Flow pressure curve of well A227X and A246X

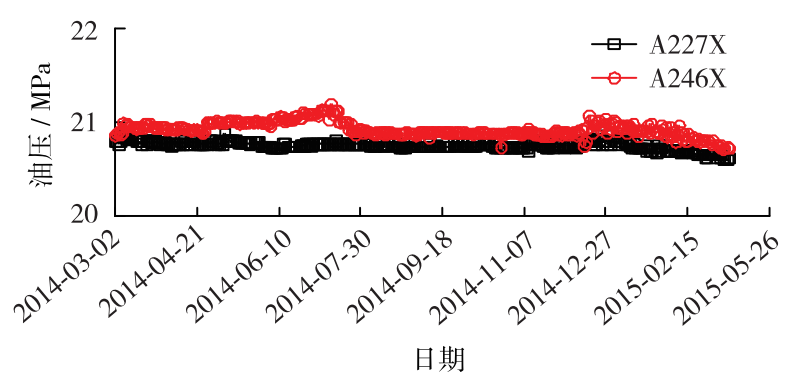

图 $7 \mathrm{A227X}$ 和 $\mathrm{A246X}$ 井油压动态曲线

Fig. 7 (Color online) Tubing pressure curve of well A227X and A246X

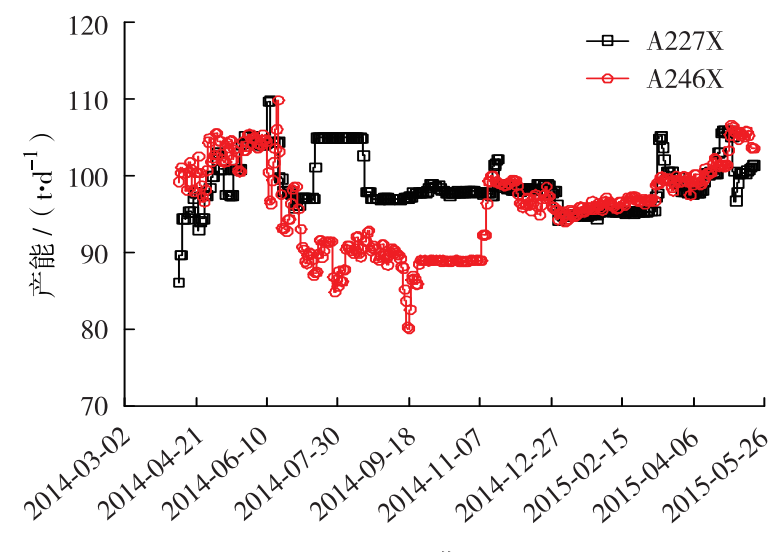

日期

图 $8 \mathrm{A227X}$ 和 $\mathrm{A246X}$ 井产能动态曲线

Fig. 8 (Color online) Oil capacity curve of well A227X and A246X

\section{3 结 论}

1) 以采油井为研究对象, 从动态分析出发优 选出油压、流压、产能和动液面 4 类生产动态数据, 基于 DTW 算法设计井间连通自动识别方法, 并以 井间动态曲线相似度为基础，采用 “五标度层次分
析-熵权法”计算各动态曲线相似度指标的权重, 建立了天然能量开发的缝洞型油藏的采油井间连通 程度评价模型，结合矿场实例对评价方法的准确性 进行了验证, 结果表明: 评价模型计算结果与静态 地质连通性和生产动态特征变化具有较高一致性.

2) 在进行评价指标权重计算时, 采用 “五标 度层次分析法” 的赋值原则确定评价指标比较矩 阵, 求解指标权重, 并采用 “熵权法” 对指标权重 进行修正得到最终评价指标权重, 该方法能够有效 降低主观选择错误对指标权重产生的影响, 增加指 标权重计算的准确性.

基金项目: 陕西省重点研发计划资助项目（2019ZDLGY11-04）

作者简介: 苏泽中 (1990-), 西安石油大学博士研究生. 研究方 向: 油气田开发工程. E-mail: szz_xsyu@126.com

引文: 苏泽中, 林加恩, 柏明星, 等. 天然能量开发阶段的缝 洞型油藏井间连通性分析 $[\mathrm{J}]$. 深圳大学学报理工版, $2020,37(6)$ : 645-652.

\section{参考文献／References:}

[ 1 ] 荣元帅. 基于示踪技术的缝洞型油藏井间连通结构识 别与表征研究 [D]. 成都: 西南石油大学, 2016.

RONG Yuanshuai. Connectivity identification and characterization of well connectivity in fracture-cavity reservoir using tracer method [D]. Chengdu: Southwest Petroleum University, 2016. (in Chinese)

[ 2 ] 苏泽中. 利用动态资料研究碳酸盐岩缝洞型油藏井间 连通性 [D]. 西安: 西安石油大学, 2018.

SU Zezhong. The study of inter-well connectivity in fracture-vuggy carbonate reservoirs by dynamic data $[\mathrm{D}]$. Xi'an: Xi'an Shiyou University, 2018. (in Chinese)

[ 3 ] 胡广杰. 塔河油田缝洞型油藏周期注水开发技术政策 研究 $[\mathrm{J}]$. 新疆石油地质, $2014,35(1)$ : 59-62.

HU Guangjie. Technological policy research of cyclic waterflooding in fracture-cavity carbonate reservoir in Tahe oilfield [J]. Xinjiang Petroleum Geology, 2014, 35(1) : 59-62. (in Chinese)

[4] 张新宝, 李留仁. 碳酸盐岩缝洞型油藏井间连通性的 示踪剂监测——塔河油田 T402 注采井组为例 $[\mathrm{J}]$. 西安石油大学学报自然科学版, 2019, 34(6) : 55-59.

ZHANG Xinbao, LI Liuren. Tracer monitoring of interwell connectivity in fracture-vuggy carbonate reservoir: a case study of T402 injection-production well group in Tahe oilfield $[\mathrm{J}]$. Journal of Xi'an Shiyou University Natural Science Edition, 2019, 34(6) : 55-59. (in Chinese)

[ 5 ] 曹 新, 王林杰, 于兆坤. 示踪剂监测技术在储层均质 性和油层连通性中的应用 $[\mathrm{J}]$. 非常规油气, 2019, 6 (3) : 71-76.

CAO Xin, WANG Linjie, YU Zhaokun. Application of tracer monitoring technology in reservoir homogeneity and reservoir connectivity $[\mathrm{J}]$. Unconventonal Oil \& Gas, 
2019, 6(3): 71-76. (in Chinese)

[6] 刘振宇, 曾昭英, 翟云芳, 等. 利用脉冲试井方法研究 低渗透油藏的连通性 $[\mathrm{J}]$. 石油学报, 2003, 24 (1)： 73-77.

LIU Zhenyu, ZENG Zhaoying, ZHAI Yunfang, et al. Study on connectivity of low permeability reservoirs with pulse-testing method $[\mathrm{J}]$. Acta Petrolei Sinica, 2003, 24 (1) : 73-77. (in Chinese)

[7]易 斌, 崔文涁, 鲁新便, 等. 塔河油田碳酸盐岩缝洞 型储集体动态连通性分析 $[\mathrm{J}]$. 新疆石油地质, 2011, $32(5): 469-472$.

YI Bin, CUI Wenbin, LU Xinbian, et al. Analysis of dynamic connectivity on carbonate reservoir with fracture and cave in Tahe field, Tarim Basin $[\mathrm{J}]$. Xinjiang Petroleum Geology, 2011, 32(5) : 469-472. (in Chinese)

[8] 彭琼玉. 塔河缝洞型油藏井间连通性研究 [D]. 成都: 西南石油大学, 2019.

PENG Qiongyu. Study on interwell connectivity of Tahe fracture-cavity reservoir [D]. Chengdu: Southwest Petroleum University, 2019. (in Chinese)

[ 9 ] 谢昕翰, 闰长辉, 赖思宇, 等. 塔河六区缝洞型碳酸盐 岩油藏井间连通类型研究 $[\mathrm{J}]$. 科学技术与工程, $2013,13(34)$ : 10284-10288

XIE Xinhan, YAN Changhui, LAI Siyu, et al. Inter-well connectivity types analysis of fracture-cavity carbonate reservoir at block 6 in the Tahe oil fields $[\mathrm{J}]$. Science Technology and Engineering, 2013, 13 (34) : 10284-10288. (in Chinese)

[10] 杨小峰, 康志宏, 陈 夷, 等. 塔河油田 6 区、7 区岩 溶型碳酸盐岩油藏动态连通性及缝洞单元划分 $[\mathrm{J}]$. 科学技术与工程, $2015,15(1)$ : 182-188.

YANG Xiaofeng, KANG Zhihong, CHEN Yi, et al. Dynamic connectivity and fracture-vuggy units division of karst carbonate reservoir in block 6-7 of Tahe oilfield [J]. Science Technology and Engineering, 2015, 15(1) : 182-188. 5. (in Chinese)

[11] 康志宏, 陈 琳, 鲁新便, 等. 塔河岩溶型碳酸盐岩缝 洞系统流体动态连通性研究 $[\mathrm{J}]$. 地学前缘, 2012, 19 (2) : 110-120.

KANG Zhihong, CHEN Lin, LU Xinbian, et al. Fluid dynamic connectivity of karst carbonate reservoir with fracture \& cave system in Tahe oilfield. [J]. Earth Science Frontiers, 2012, 19(2) : 110-120. (in Chinese)

[12] ALABERT F G, MODOT V. Stochastic models of reservoir heterogeneity: impact on connectivity and average permeabilities [ C ]// SPE Annual Technical Conference and Exhibition. Washington D C: Society of Petroleum Engineers, 1992: 355-370.

[13 ] ALBERTONI A, LAKE L W. Inferring interwell connectivity only from Well-Rate fluctuations in waterfloods $[\mathrm{J}]$. SPE Reseroior Evaluation \& Engineering, 2003, 6(1): 6-16.
[14] YOUSEF A A, Gentil P, JENSEN J L. A capacitance model to infer interwell connectivity from production and injection rate fluctuations $[\mathrm{J}]$. SPE Journal, 2003, 9 (6) : 636-646.

[15] 赵 辉, 康志江, 张 允, 等. 表征井间地层参数及油 水动态的连通性计算方法 $[\mathrm{J}]$. 石油学报, 2014, 35 (5) : 922-927.

ZHAO Hui, KANG Zhijiang, ZHANG Yun, et al. An interwell connectivity numerical method for geological parameter characterization and oil-water two-phase dynamic prediction [J]. Acta Petrolei Sinica, 2014, 35(5) : 922927. (in Chinese)

[16] 赵 辉, 谢鹏飞, 曹 琳, 等. 基于井间连通性的油藏 开发生产优化方法 [J]. 石油学报, 2017, 38(5) : 555561.

ZHAO Hui, XIE Pengfei, CAO Lin, et al. Reservoir production optimization method based on inter-well connectivity $[\mathrm{J}]$. Acta Petrolei Sinica, 2017, 38(5) : 555-561. (in Chinese)

[17] 赵艳艳. 缝洞型油藏井间连通程度定量评价方法及其 应用 $[\mathrm{J}]$. 西安石油大学学报自然科学版, 2017, 32 (3) : 68-72.

ZHAO Yanyan. Quantitative evaluation method of interwell connectivity for fractured-vuggy reservoirs and its application $[\mathrm{J}]$. Journal of Xi'an Shiyou University Natural Science Edition, 2017, 32(3) : 68-72. (in Chinese)

[18］田英冬, 张 元, 韩 学. 基于动态时间规整算法的虚 拟沙画手势识别方法 $[\mathrm{J}]$. 中国科技论文，2017，12 (20) : 2361-2367.

TIAN Yingdong, ZHANG Yuan, HAN Xie. Method for recognizing gesture of virtual sand painting based on dynamic time warping algorithm $[\mathrm{J}]$. China Science Paper, 2009, 2017, 12(20): 2361-2367. (in Chinese)

[19] 王 霞, 段庆全. 基于改进层次分析法的油气管道风 险因素权重计算 $[J]$. 油气储运, 2019, 38(11)：12271231.

WANG Xia, DUAN Qingquan. A method for calculating the weight of oil and gas pipeline risk factor based on improved analytic hierarchy process $[\mathrm{J}]$. Oil \& Gas Storage and Transportation, 2019, 38 (11): 1227-1231. (in Chinese)

[20] 宋明水, 刘 振, 张学才, 等. 基于改进层次熵分析法 的致密砂岩储层可压性评价一以准噶尔盆地 Z109 井侏罗系储层为例 $[\mathrm{J}]$. 地质力学学报, 2019, 25(4)： 509-517.

SONG Mingshui, LIU Zhen, ZHANG Xuecai, et al. Fracability evaluation of tight reservoirs based on improved entropy analytic hierarchy process: taking the Jurassic reservoirs of well Z109 in the Junggar Basin as an example [J]. Journal of Geomechanics, 2019, $25(4)$ : 509-517. (in Chinese)

【中文责编：晨 兮; 英文责编：天 澜】 